\title{
Alteration in the center of mass trajectory of patients after stroke
}

\author{
Aline Araujo do Carmo $^{1,2}$, Ana Francisca Rozin Kleiner ${ }^{1,3}$, Ricardo M. L. Barros \\ ${ }^{1}$ Laboratory of Instrumentation for Biomechanics, Faculty of Physical Education, University of Campinas-UNICAMP, \\ Brazil, ${ }^{2}$ Faculty of Ceilandia, FCE, University of Brasilia-UnB, Brazil, ${ }^{3}$ CAPES Foundation, Ministry of Education \\ of Brazil, Brazil
}

Background: The movement disorders due to stroke can alter the motion of the Center of Mass (CoM) of the body. Thus, the analysis of the CoM motion can be an alternative to diagnostic the stroke gait disturbances and has not been widely explored.

Objective: To identify and to analyze the alterations of CoM trajectory during both of gait cycles, affected and unaffected, of post-stroke patients comparing to healthy subjects.

Methods: The CoM trajectory was obtained using a gold standard method, the three-dimensional (3D) kinematics associate to anthropometry. Two experimental groups were compared: Hemiparetic Group (HG) consisted of fourteen chronic hemiparetic patients and Control Group (CG) by fourteen able-bodied subjects. Results: The statistical analysis $(P \leq 0.05)$ revealed the following average gait alterations in the $\mathrm{HG}$, in the stance phase of the affected side: higher lateral (midstance), lower vertical (midstance and terminal stance), and lower forward displacement (heel strike until terminal stance). In the swing phase of the affected side, HG showed higher lateral (preswing and initial swing) and vertical displacement (preswing until terminal swing), and lower forward (preswing until terminal swing) displacement of the CoM. There was also anticipation of the instants of maximum displacements in the lateral and vertical directions and lower total range in the forward direction.

Conclusion: The CoM trajectory analysis pointed out that the gait after stroke was altered such in the affected as in the unaffected lower limbs, mainly in the single support phase of the affected side, but also in the swing phase of the gait cycle.

Keywords: Stroke, Center of mass, Gait analysis, Biomechanics, Walking

\section{Introduction}

The central nervous system disorders, like stroke, affecting the pyramidal or extrapyramidal systems and musculoskeletal disorders, disturb normal movements of the body segments during gait. ${ }^{1}$ Hemiplegia due to stroke significantly limits gait performance. Hemiplegic gait is characterized as slow, stiff, and poorly coordinated movements of the affected leg and compensatory movements. Reduction in gait speed, asymmetry of gait, impairment in kinematics and kinetics, abnormality in electromyographic patterns, increase in energy cost, and reduction in ambulatory activity have all been reported. ${ }^{2-6}$ These movement disorders may lead alterations in the motion of the center of mass of the body and for this reason can constitute an important and different way to analyze the pathological gait. ${ }^{7}$

Correspondence to: A. A. do Carmo, Rua 37 sul, Lote 16, Residencial Rivoli, apartamento 607, CEP 71931-540, Brazil. Email: aline.acarmo@ ig.com.br
Several methods exist for the estimation of Center of Mass $(\mathrm{CoM})$ movement that differs in the underlying assumptions. The segmental kinematics method is based on the definition of the CoM by measuring the position and orientation of each segment and approximating the mass fractions as well as the locations of the CoM of each segment then, an estimation of the overall CoM movement is obtained. ${ }^{8}$ The performance of the segmental kinematics method requires a precise marker set for accurate position and orientation of the body segments. The most common method integrates the three components of the ground reaction force. However, this requires a subject to hit force plates, which can be difficult for those with a pathological gait pattern. Furthermore, this method provides the CoM displacement, but not the absolute threedimensional (3D) position in space because of the absence of the initial constants required in the double integration, the initial position and initial velocity. ${ }^{8-12}$ Other least accurate methods of estimating CoM were 
found, such as the use of a single anatomical landmark. ${ }^{13}$ However, comparing methods of estimating the total body CoM showed that for an accurate determination of the position of the CoM a full body segmental kinematics method is required. ${ }^{14}$

The movement of the CoM in gait represents the overall effects of joint and segment kinematics during forward progression. It has therefore been used as a parameter in gait analysis by a number of studies investigating patients with various pathologies. ${ }^{2,7,13,15}$ However, the most of researches using the CoM are focused on the mechanical energy cost ${ }^{11,12,15-20}$ and the CoM trajectory has not been widely explored. The analysis of the CoM trajectory continuously during walking contains important information about dynamic balance control and especially for pathological gait which the CoM movement can vary from step to step. ${ }^{8}$ The findings from CoM trajectory analysis can be complementary to the traditional gait analysis exams and helpful to better understand the biomechanics of stroke gait.

In the post-stroke gait some studies used the CoM analysis. A new rehabilitation strategy to reduce the walking energy costs in hemiparetic patients by helping them to actively reduce excessive vertical CoM displacement through biofeedback showed positive results reducing energy cost after intervention and found that the energy cost decreased $30 \%$ in those patients. $^{15}$ The vertical excursion of the CoM during walking was measured in the stance phase of each limb in order to evaluate the compensatory contributions of the contra-lateral limb, their results showed asymmetric vertical excursion of the CoM and compensatory elevation of the CoM in the stance phase of the contra-lateral limb. ${ }^{13}$

However, to the best of our knowledge, no further study compared inter and intra-cycle variables of affected, unaffected side of post-stroke subjects to the CoM trajectory of normal subjects in order to identify the specific alterations produced in the gait after stroke, using a full body kinematic approach. Thus, the aim of this work was to identify and to analyze the alterations of center of mass trajectory during both of gait cycles, affected and unaffected, of post-stroke patients comparing to healthy subjects.

\section{Methods}

This research was approved by the University of Campinas Ethics Research Committee (process number 789/2007). Volunteers were informed about all procedures and signed an informed consent.

\section{Subjects}

Twenty eight subjects were analyzed in this study. The hemiparetic group (HG) was composed of 14 individuals affected by stroke as presented on Table 1. The inclusion criteria were: male subject, affected by only one stroke, aged between 40 and 60 years, at least 3 years after stroke and without using any assistive device at the time of data collection. Impairment level of $\mathrm{HG}$ was also assessed by means of clinical rating scales in order to characterize the patients. In Fugl-Meyer ${ }^{21}$ motor scale, the subjects reached average score of $49.3 \pm 20.7$, indicating considerable motor impairment. In the Berg balance scale, ${ }^{22}$ the $\mathrm{HG}$ average score was 50, indicating slightly altered functional balance. The modified Ashworth Scale ${ }^{23}$ classified the majority of the muscle groups as Grade 1, indicating mild hypertonia of both the upper and lower limbs. Moreover, the subjects were asked about the handedness and all subjects were right-handed.

The control group (CG) consisted of 14 male healthy adults (age: $51.0 \pm 5.0$ years old; body mass: $82.4 \pm 11.7 \mathrm{~kg}$; height: $1.69 \pm 0.05$ meters). The nondisabled controls exhibited normal joint range of motion and muscle strength and had no gait abnormalities.

\section{Kinematic analysis system}

The DVideo kinematic analysis system ${ }^{24-26}$ was used to obtain the $3 \mathrm{D}$ coordinates of the markers. The system consisted of four genlocked Basler cameras (Model A602fc) working at a 75-Hz frame rate.

\section{Kinematic and anthropometrical model}

The protocol for body segment position and orientation (Fig. 1) considers the human body as a system with 13 ball-and-socket joints with $3^{\circ}$ of freedom in each joint. $^{27}$ The modeled segments were the pelvis, scapulas, arms, forearms, thighs, shanks, and feet. For orientation purposes, surface markers, and technical markers were fixed on the subject's skin. In a static trial, the subjects used both sets of markers. During the dynamic trial, only the technical marker set remained, which reduced the number of markers during the execution of movements and facilitated the identification of markers of the kinematic analysis system. The markers were positioned as shown in Fig. 1. The hip and the glenohumeral joint centers were calculated according to prediction method. ${ }^{28,29}$ The ankle, knee, elbow, and wrist joint centers were calculated as the midpoint between the lateral and medial markers in the static trial.

An anthropometric model ${ }^{30,31}$ was applied in order to obtain the inertial parameters needed to the CoM calculation. The anatomical length and circumference 
Table 1 Hemiparetic group characteristics

\begin{tabular}{|c|c|c|c|c|c|c|c|c|}
\hline Subject & Height (m) & $\begin{array}{l}\text { Body mass } \\
(\mathrm{kg})\end{array}$ & $\begin{array}{c}\text { Age } \\
\text { (years) }\end{array}$ & $\begin{array}{c}\text { Time since } \\
\text { stroke (years) }\end{array}$ & $\begin{array}{l}\text { Affected } \\
\text { Side }\end{array}$ & $\begin{array}{l}\text { Fugl-Meyer } \\
\text { Score }\end{array}$ & $\begin{array}{c}\text { Berg Balance } \\
\text { Score }\end{array}$ & Ashworth \\
\hline $\mathrm{H}_{1}$ & 1.66 & 49.5 & 47.0 & 9 & $\mathrm{R}$ & 61 & 52 & 1 \\
\hline $\mathrm{H}_{2}$ & 1.76 & 92.0 & 45.0 & 7 & $\mathrm{R}$ & 30 & 46 & $1+$ \\
\hline $\mathrm{H}_{3}$ & 1.72 & 99.5 & 70.0 & 15 & $\mathrm{~L}$ & 15 & 46 & 2 \\
\hline $\mathrm{H}_{4}$ & 1.75 & 83.7 & 35.0 & 10 & $L$ & 54 & 56 & 2 \\
\hline $\mathrm{H}_{5}$ & 1.62 & 79.5 & 68.0 & 15 & $L$ & 65 & 50 & 1 \\
\hline $\mathrm{H}_{6}$ & 1.63 & 66.0 & 57.0 & 9 & $\mathrm{R}$ & 54 & 46 & $1+$ \\
\hline $\mathrm{H}_{7}$ & 1.74 & 67.0 & 52.0 & 10 & $\mathrm{R}$ & 50 & 56 & 1 \\
\hline $\mathrm{H}_{8}$ & 1.63 & 76.3 & 62.0 & 5 & $\mathrm{~L}$ & 27 & 37 & 1 \\
\hline $\mathrm{H}_{9}$ & 1.60 & 81.8 & 60.0 & 5 & $\mathrm{R}$ & 35 & 49 & $1+$ \\
\hline $\mathrm{H}_{10}$ & 1.72 & 71.6 & 48.0 & 9 & $\mathrm{~L}$ & 35 & 52 & 2 \\
\hline $\mathrm{H}_{11}$ & 1.63 & 59.2 & 53.0 & 7 & $\mathrm{R}$ & 72 & 52 & 1 \\
\hline $\mathrm{H}_{12}$ & 1.60 & 68.6 & 49.0 & 3 & $\mathrm{R}$ & 87 & 56 & 0 \\
\hline $\mathrm{H}_{13}$ & 1.71 & 90.0 & 59.0 & 4 & $\mathrm{R}$ & 34 & 52 & 2 \\
\hline $\mathrm{H}_{14}$ & 1.74 & 64.6 & 38.0 & 4 & $\mathrm{~L}$ & 72 & 52 & 1 \\
\hline Mean $\pm S D$ & $1.68 \pm 0.06$ & $74.9 \pm 13.7$ & $53.0 \pm 10.3$ & $8.0 \pm 3.8$ & $\ldots$ & $49.3 \pm 20.7$ & $50 \pm 5$ & $\ldots$ \\
\hline
\end{tabular}

Note: $S D=$ standard deviation; $R=$ right; $L=$ left.

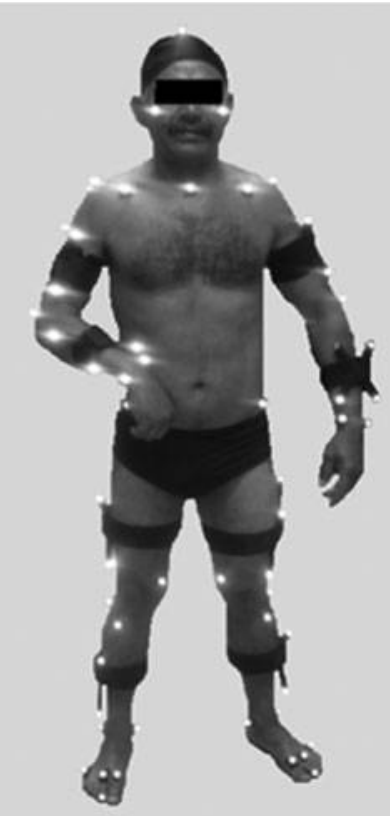

(a)

Figure 1 Representation of the experimental protocol, anthropometrical model, and the stick figure results of a normal subject. (A) An anterior view of the protocol for gait analysis. (B) The joint centers and the body segmentation (Zatsziorsky et al., 1990; de Leva et al., 1996): 1, vertex of parietal bone; 2, supraesternale; 3, left glenohumeral joint center; 4, left elbow joint center; 5, left wrist joint center; 6, right hip joint center; 7, left hip joint center; 8, mid-hip; 9, left knee joint center; 10, left ankle joint center; and 11, left tip-toe. (C) An illustration of the center of mass of each segment (asterisks) and the total body center of mass trajectory (line).

of each body segment were measured to obtain the relative body segments' mass. ${ }^{31}$

\section{Experimental procedures}

After the markers placement the subjects were asked to walking for some minutes in order to get used to the laboratory environment, then each participant was asked to walk barefoot, at a comfortable selfselected gait speed, without any assistive device (canes or orthosis). One gait cycle of the right limb and one gait cycle of the left limb were collected for the $\mathrm{HG}$ and $\mathrm{CG}$ subjects. However, as in the $\mathrm{HG}$, there were seven subjects affected by right side hemiparesis and seven subjects affected by left side hemiparesis, the gait cycles were separated in affected cycle and unaffected cycle.

\section{Center of mass calculation}

The 3D coordinates of the points were used to determine the joint centers. According to the model ${ }^{30,31}$ for the kinematic estimation of the CoM, the body segments are defined by a vector between two 
adjacent joint centers. ${ }^{29}$ The total body CoM was obtained by the follow equation (1):

$$
\vec{r}_{\mathrm{CM}}=\frac{\sum_{\mathrm{i}=1}^{\mathrm{N}} m_{\mathrm{i}} \vec{r}_{\mathrm{i}}}{\sum_{\mathrm{i}=1}^{\mathrm{N}} m_{\mathrm{i}}}
$$

where $m_{\mathrm{i}}$ is the relative mass of each body segment and $\vec{r}_{\mathrm{i}}$ is the position vector of the $i$ th body segment.

\section{Data processing}

The raw data were filtered with a zero-phase forward and reverse 4th-order Butterworth digital filter with a $6-\mathrm{Hz}$ cut-off frequency. All the CoM curves were represented as a function of the percentage of gait cycle and centered in the mean value to eliminate the effect of the absolute position of the subject in the global coordinate system.

\section{Experimental variables}

Two kinds of variables were analyzed for both groups HG and CG: continuous and discrete. The continuous variables were the CoM trajectory in the lateral, vertical and forward directions. The discrete variables were extracted from CoM curves in lateral and vertical directions (Fig. 2). The CoM trajectory during gait is approximately sinusoidal in the lateral and vertical directions. ${ }^{32}$ Thus, in order to analyze the CoM motion in the stroke gait, the following discrete experimental variables were obtained:

1. the values of maximum displacement in the stance and swing phases in lateral (MD_STlateral; MD_SWlateral) and vertical (MD_STvertical; MD_SWvertical) directions;

2. difference between maximum displacement in stance and swing phase (DST_SW) for both lateral and vertical directions;

3. percent of location in the gait cycle of the maximum displacement in the stance and swing phases for both lateral and vertical directions (\%MD_STlateral; \%MD_SWlateral; \%MD_STvertical, and \%MD_SWvertical);

4. total ranges (TR) of trajectory curves in vertical, lateral and forward directions;

\section{Statistical analysis}

The statistical analysis was performed with Matlab $^{\circledast}$ software. Before of any comparison, normality (Lilliefors test, $P \leq 0.05)$ and variance ( $F$-test, $P \leq 0.05)$ were tested. When normal distribution and equality of variances were found, parametric analysis using Student's $t$-test $(P \leq 0.05)$ was applied. For non-parametric statistics, the Wilcoxon test and Mann-Whitney $U$ test $(P \leq 0.05)$ were applied.

No significant difference was found in any variables $(P \leq 0.05)$ between CG left- and right-cycle movements. Then, considering that CG subjects were right-handed, only the CG's right gait cycle results were used for all comparisons between groups.
The statistical comparisons were divided in intragroup and intergroup. The intragroup comparison was performed between $\mathrm{HG}$ affected side versus HG unaffected side. The intergroup comparison was performed between CG right gait cycle versus $\mathrm{HG}$ affected side and also unaffected side.

The continuous curves of the CoM trajectory of HG and CG were also compared by applying statistical tests to each percent of the hundred-percent gait cycle to identify group's differences in intracycle phases.

\section{Results}

No statistical difference was found comparing the hemiparetic and control groups for age $(P=0.318)$, weight $(P=0.654)$, and height $(P=0.232)$.

\section{Discrete variables}

Table 2 shows the statistical analysis comparing mean and standard deviation of the maximum displacement in stance phase (MD_ST) and maximum displacement in swing phase (MD_SW), difference between MD_ST and MD_SW and range of the CoM trajectory in the vertical and lateral directions.

The HG affected side compared to CG presented difference for the MD_SWvertical $(P=0.042)$ and total range forward $(P=0.013)$, while the unaffected side showed differences for MD_STlateral $(P=0.031)$, MD_STvertical $(P=0.002)$, MD_SWvertical $(P=0.004)$, and range forward $(P<0.001)$.

Comparing $\mathrm{HG}$ and $\mathrm{CG}$, no statistical difference was found between affected side and CG in lateral displacement in the stance $(P=0.1707)$ or swing $(\mathrm{P}=0.1582)$ phase as well as in the vertical direction in stance phase $(P=0.4645)$.

For both affected and unaffected sides of HG, significant differences were found between support and swing phases, MD_STlateral $(P=0.003 ; P=0.026)$ and MD_SWlateral $(P=0.004 ; P=0.027)$, and also between MD_STvertical $(P=0.004 ; P<0.001)$ and MD_SWvertical $(P=0.004 ; P<0.001)$.

The average percent of location in the gait cycle of the maximum displacements values showed significant differences when the HG affected side was compared to CG. The affected side showed anticipation of the \%MD_STlateral $(\mathrm{HG}=30.7 \%, \mathrm{CG}=41.7 \%$, $P=0.0063), \quad \% \mathrm{MD} \_S T$ vertical $\quad(\mathrm{HG}=25.9 \%$, $\mathrm{CG}=31.3 \%, \quad P=0.0132$ ), and \%MD_SWvertical $(\mathrm{HG}=74.4, \quad \mathrm{CG}=80.3, \quad P=0.0135)$. No significant difference was found comparing the $\mathrm{HG}$ unaffected side to CG. However, the comparison of the affected side showed anticipation of the \%MD_SWlateral $(\mathrm{AF}=80.4 \%, \mathrm{UF}=88.5 \%, P=0.0034), \% \mathrm{MD} \_\mathrm{ST}$ Tertical 
Table 2 Mean and Standard deviation of the variables extracted from center of mass trajectory in lateral and vertical directions

\begin{tabular}{|c|c|c|c|c|c|c|}
\hline \multirow[b]{2}{*}{ Variables } & \multicolumn{3}{|c|}{ Lateral (cm) } & \multicolumn{3}{|c|}{ Vertical (cm) } \\
\hline & AF & UF & CG & AF & UF & CG \\
\hline MD_ST & $4.2(2.2)^{\delta}$ & $3.8(1.4)^{\delta}$ & $2.9(0.9)^{\boldsymbol{\Lambda}}$ & $0.9(0.8)^{*}$ & $1.8(0.7)^{* \boldsymbol{\Lambda}}$ & $1.2(0.3)^{\boldsymbol{\Lambda}}$ \\
\hline MD_SW & $3.6(2.2)^{\delta}$ & $4.5(2.2)^{\mathbf{\Lambda} \delta}$ & $2.4(1.1)$ & $1.9(0.7) \dagger^{*}$ & $0.2(0.7)^{* \boldsymbol{\Lambda}}$ & $0.8(0.4)^{\wedge}$ \\
\hline DST_SW & $0.7(0.5)$ & $1.1(0.6)$ & $0.7(0.6)$ & $0.8(0.8)$ & $1.2(0.9)^{\mathbf{\Lambda}}$ & $0.4(0.2)^{\boldsymbol{\Lambda}}$ \\
\hline TR & $8.9(3.8)$ & $9.3(3.2)$ & $7.2(1.3)$ & $3.9(1.2)$ & $3.8(1.1)$ & $2.9(0.7)$ \\
\hline \%MD_ST & $30.7(7.0) \dagger$ & $36.6(10.4)$ & $41.7(5.3) \dagger$ & $25.9(5.3) \dagger^{\gamma}$ & $31.1(4.8)^{\gamma}$ & $31.3(2.6) \dagger$ \\
\hline \%MD_SW & $80.4(7.3)^{\gamma}$ & $88.5(6.3)^{\gamma}$ & $83.0(8.5)$ & $74.4(6.5) \dagger^{\gamma}$ & $83.8(4.4)^{\gamma}$ & $80.3(3.5) \dagger$ \\
\hline
\end{tabular}

Note: MD_ST=maximum displacement in stance phase; MD_SW=maximum displacement in swing phase; \%MD_ST and \%MD_SW=Percent value corresponding to the instant which the maximum displacement in the stance phase and swing phase occurred in the gait cycle; DST_SW=difference between MD_ST and MD_SW; Total range (TR) of the center of mass trajectory in the vertical and lateral directions.

${ }^{\delta}$ Significant differences between MD_ST and MD_SW for lateral direction $P \leq 0.05$.

* Significant differences between MD_ST and MD_SW for vertical direction $P \leq 0.05$. $\dagger$ Significant differences between Affected side and $\mathrm{CG} P \leq 0.05$

$\Delta$ Significant differences between Unaffected side and CG $P \leq 0.05 .{ }^{\gamma}$ Significant differences between Affected and Unaffected sides $P \leq 0.05$
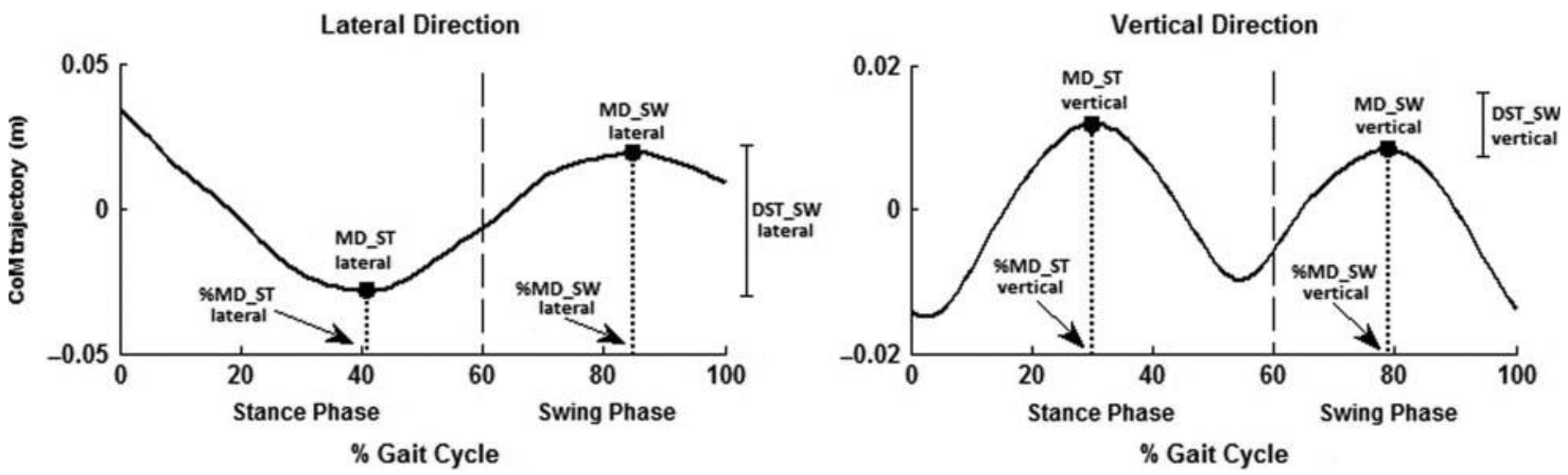

Figure 2 Illustration and definition of the discrete variables extracted from CoM trajectories of lateral and vertical direction. A curve of one gait cycle of control group subject was used as example. MD_ST = maximum displacement in stance phase; MD_SW = maximum displacement in swing phase; \%MD_ST and \%MD_SW $=$ Percent value corresponding to the instant which the maximum displacement in the stance phase and swing phase occurred in the gait cycle; DST_SW = difference between MD_ST and MD_SW.

$(\mathrm{AF}=25.9 \%, \quad \mathrm{UF}=31.1 \%, \quad P=0.0127), \quad$ and $\% \mathrm{MD}$ SWvertical $(\mathrm{AF}=74.4 \%, \quad \mathrm{UF}=83.8 \%, P \leq 0.001)$ to the unaffected side.

\section{Continuous variables: CoM trajectories}

Figure 3 shows the average CoM trajectory in lateral, vertical, and forward directions, during affected and unaffected sides of the $\mathrm{HG}$ subjects and a right cycle of the $\mathrm{CG}$ subjects. Moreover, statistical analysis along the gait cycle allowed the identification of the phases where the CoM trajectory of HG were significantly different $(P \leq 0.05)$ from the $\mathrm{CG}$ for the three movements direction.

The average CoM curve of control group presents the expected form as shown in many studies of normal gait. In the lateral direction, positive and negative values are associated to the arbitrary definition of left (positive) and right (negative) sides. Therefore, the peak and valley form of the curve represents the absolute displacement to the left (or right) in the CoM oscillation.

In vertical direction, the normal gait curve is approximately sinusoidal with two peaks, the first one in the midstance phase and the second one in the midswing phase. The CoM forward trajectory has an increasing form from posterior (negative) to anterior (positive) position related to the average position of the CoM trajectory.

The lateral oscillation of $\mathrm{HG}$ affected side was higher than CG $(P \leq 0.05)$ from 9 to $27 \%$ of the gait cycle corresponding to the midstance phase. Differences were found also from 50 to $71 \%$, corresponding to preswing and initial swing phases. When the unaffected side cycle was compared to the $\mathrm{CG}$, the lateral CoM displacement was increased from 13 to $27 \%$ inside the midstance phase and from 75 to $99 \%$ of the cycle, in the midswing and terminal swing. 

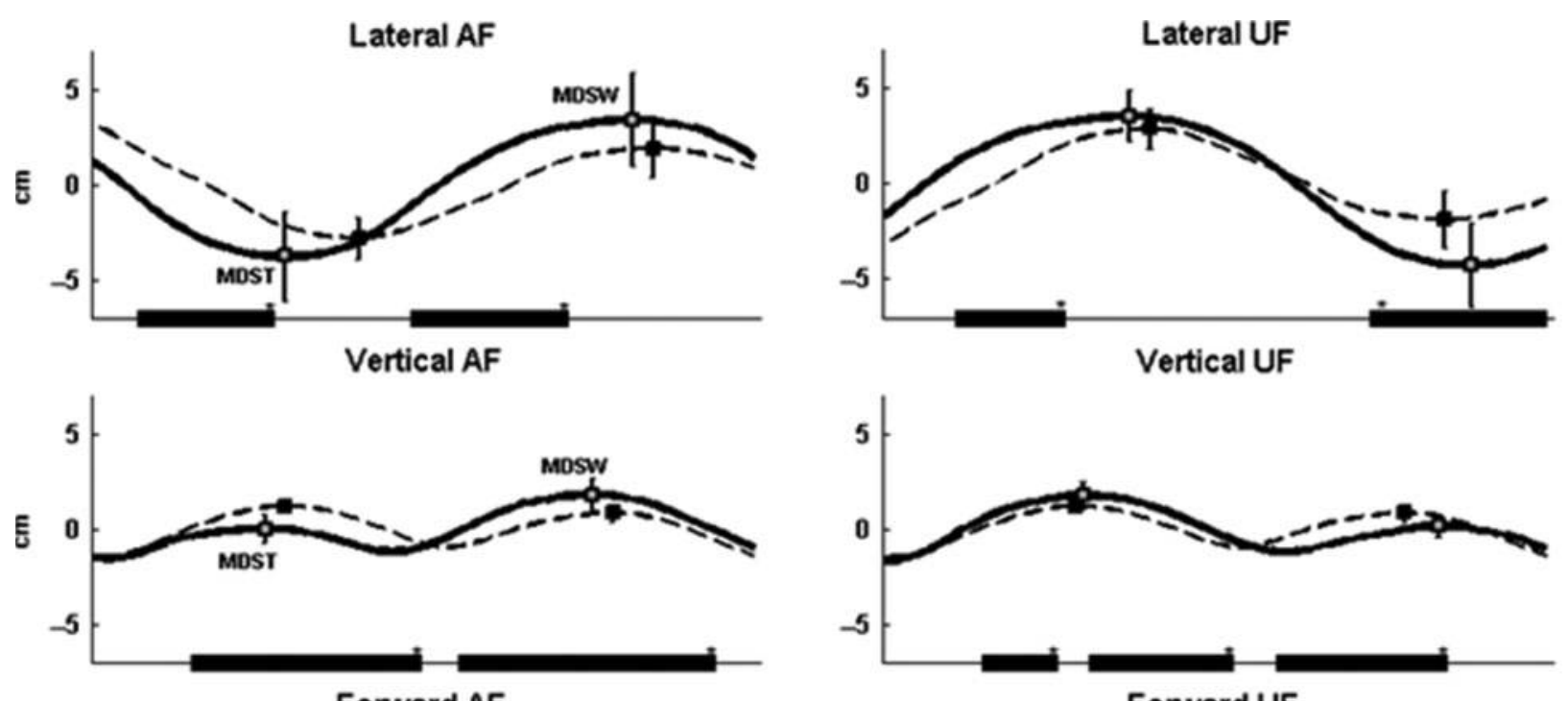

Forward AF
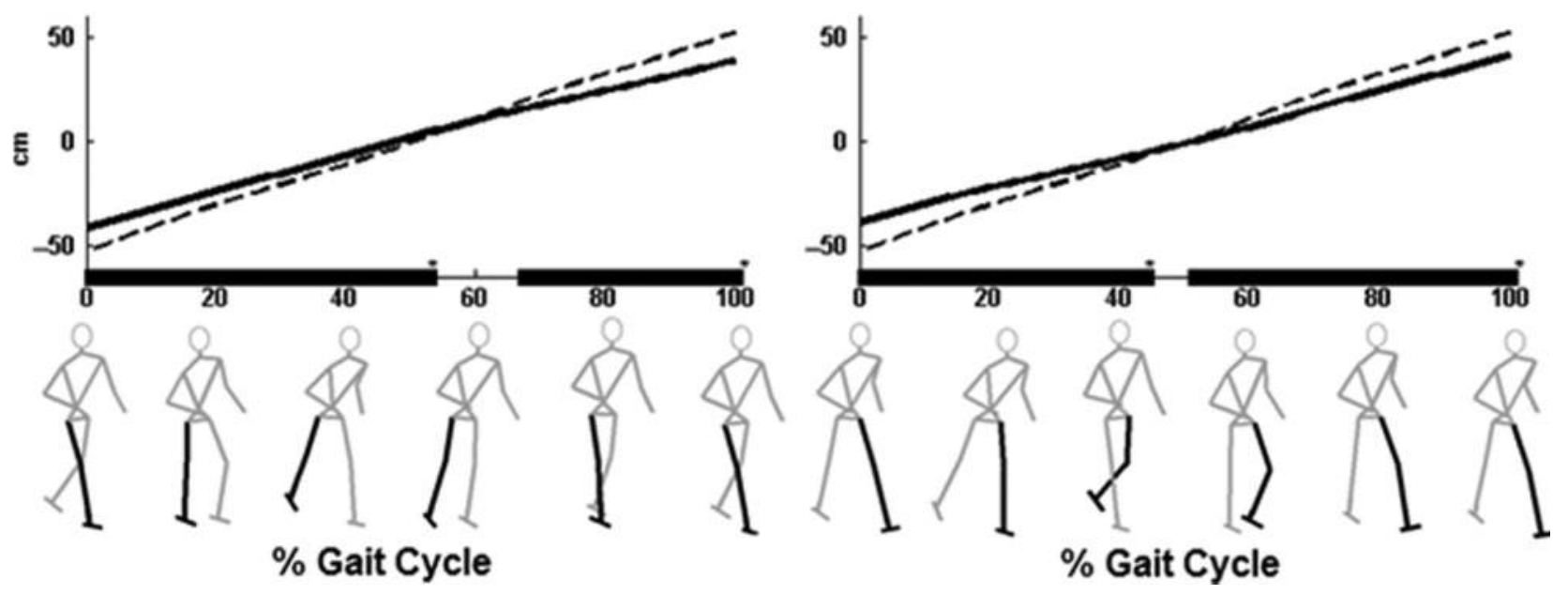

Figure 3 Average curve of the center of mass trajectory in the lateral, vertical and forward directions of the hemiparetic group (bold solid line: $n=14$ ) and the control group (bold dashed line: $n=7$ ). The columns show, respectively, the mean of center of mass trajectory in the affected cycle (AF) and unaffected cycle (UF) of the hemiparetic group. On the $x$-axes, - indicates the phases of the gait cycle that presented significant differences $(P \leq 0.05)$ between the hemiparetic and control group curves. P1 and P2 mean, respectively, the maximum displacement in stance phase and in swing phase for lateral and vertical directions.

In vertical displacement, the $\mathrm{HG}$ affected side curve is under the CG curve from $17-49 \%$ (midstance and terminal stance phases) and over the CG curve during all swing phase (57-93\%). The HG unaffected side presented approximately the opposite behavior as the affected side in vertical direction, except by the small phase from $27-32 \%$ of cycle.

In the forward direction, significant differences were observed along the cycle for both, affected (1-53 and 68-100\%) and unaffected limbs (1-44 and $52-100 \%)$, except during the double support phase $(P \leq 0.05)$. This result was expected since the HG walked slower than $\mathrm{CG}$ and, therefore, covered a smaller distance in one gait cycle.

\section{Discussion}

The aim of this study was to identify the alterations of CoM 3D trajectory in gait of post-stroke patients. It was demonstrated that the HG affected side was characterized by the following alterations in the CoM trajectory: increased lateral and reduced vertical and forward CoM displacements in the single stance phase. The swing phase was characterized by higher lateral CoM displacement at the initial swing phase, increased vertical CoM displacement, higher maximum vertical displacement and reduced forward CoM displacement during all swing phase. Moreover, there was anticipation of the maximum displacements in lateral and vertical directions for both, stance and swing phases. 
Lateral CoM oscillation was increased in HG compared to normal subjects. However, it is not possible to affirm that this alteration is a distinctive characteristic of stroke patients' gait since previous study ${ }^{33}$ observed in normal subjects' larger lateral CoM displacement at slower speeds and the walking speed was not controlled in the present study, since selfselected speed was adopted as protocol.

A remarkable finding observed in the present paper was that the CoM oscillation was symmetrical with respect to zero. This suggests that despite the hemibodies asymmetry caused by stroke, the patients were able to control the CoM trajectory maintaining a straight forward gait. The same symmetrical behavior was observed looking at the CoM oscillation of the unaffected side. In a previous study of the same research group of the present paper, ${ }^{4}$ asymmetric alterations were observed in angular kinematics of lower and upper limbs, as well as asymmetrical lateral trunk motion with respect to zero, in post-stroke patients. As the whole body CoM trajectory depends on the position of each body limb (and its mass), this may lead to the conclusion that the symmetrical behavior of CoM in lateral direction might be controlled by the asymmetrical movements of the lower and upper limbs and trunk.

Another interesting result revealed by the present study was the anticipation in terms of percentage of gait cycle of the maximal lateral displacement in stroke patients. The association of the increased lateral oscillation and the anticipation of maximal displacement suggest that stroke patients need increased lateral CoM velocity and accelerations to complete the gait cycle. The faster change of CoM lateral velocity observed in post-stroke patients could partially explain the increased energy expenditure reported in the literature. ${ }^{34}$

Vertical CoM curves showed asymmetric behavior comparing stance and swing phases. The stroke patients presented lower CoM elevation than CG when supporting their weight with the affected limb. Spasticity and muscle weakness are often pathological disturbances from stroke ${ }^{4}$ and may be the primary cause of these alterations in the CoM trajectory observed in this study.

The total range of $\mathrm{CoM}$ motion of the $\mathrm{HG}$ was lower than $\mathrm{CG}$ in the forward direction as expected since the HG subjects walked slower than CG and consequently covered smaller distance.

Thus, from a methodological perspective, the CoM trajectory analysis showed to be a suitable method to determine the gait alterations and, although requires a more complex experimental set up, can be used as a tool for stroke gait evaluation. In particular, the intra-cycle analysis (Fig. 3) associated to the analysis of the discrete variables (Table 2) taken in specific gait cycle phases showed not only that differences between hemiparetic and control groups exists but also where those differences occurs in the gait cycle.

There are some limitations in the present study. The number of patients is small and the sampling of the present study was by convenience. Thus, a randomized longitudinal trial using a larger number of patients is necessary in a future study to better understand the behavior of the CoM trajectories over time. Moreover, future study should involves the correlation between results obtained by clinical evaluation scales and kinematic alterations in the CoM trajectory to verify the advantages and disadvantages in the use of each one as an assessment tool of gait after stroke.

\section{Conclusion}

The 3D analysis of the CoM trajectory in gait of stroke patients revealed alterations in the CoM in all directions and allowed to identify the exactly phase of gait cycle that these alterations occurred. The main alterations were observed in the single support phase of the affected side, where there was higher lateral displacement, lower vertical and forward displacement. In the swing phase of the affected side, there was higher lateral and vertical displacement and also lower forward displacement. The total range of the CoM in the forward direction was lower than CG, but in the vertical and lateral directions, there are no significant alterations. The alterations found in the unaffected side may constitute a compensatory strategy to improve the gait pattern.

It was demonstrated that the CoM is a kinematic variable useful to detect gait alterations by stroke, even in the unaffected side. These data are complementary to the findings obtained by others kinematical variables like joint angles and spatiotemporal gait parameters.

\section{Acknowledgements}

We acknowledge financial support from São Paulo Research Foundation-FAPESP (06/05234-6), National Council for Scientific and Technological Development CNPq (473729/2008-3; 304975/2009-5; 140731/2011-3; 306996/2013-8), and CAPES (BEX 11241/13-6). The sponsors had no role in the study design, in the collection, analysis, and interpretation of the data; in the writing of the manuscript; and in the decision to submit the manuscript for publication.

\section{Disclaimer Statements}

Contributors All author contributed equally. 
Funding Paulo Research Foundation-FAPESP (06/ 05234-6), National Council for Scientific and Technological Development - CNPq (473729/2008-3; 304975/ 2009-5; 140731/2011-3; 306996/2013-8) and CAPES (BEX 11241/13-6).

Conflicts of interest There are no conflicts of interest.

Ethics approval This research was approved by the University of Campinas Ethics Research Committee (process number 789/2007). Volunteers were informed about all procedures and signed an informed consent.

\section{References}

-1 Hahn ME, Chou L. Can motion of individual body segments identify dynamic instability in the elderly? Clin Biomech. 2003;18(1):737-744.

- 2 Kobayashi T, Leung AKL, Akazawa Y, Hutchins SW. Effect of ankle-foot orthoses on the sagittal plane displacement of the center of mass in patients with stroke hemiplegia: a pilot study. Top Stroke Rehabil. 2012;19(4):338-344.

3 Olney SJ, Richards C. Hemiparetic gait following stroke. Part I: Characteristics. Gait Posture. 1996;4(2):136-148.

-4 Carmo AA, Kleiner AFR, Lobo da Costa PH, Barros RML. Three-dimensional kinematic analysis of upper and lower limb motion during gait of post-stroke patients. Braz J Med Biol Res. 2012;45(6):537-545.

$\checkmark 5$ Michael K, Macko RF. Ambulatory activity intensity profiles, fitness, and fatigue in chronic stroke. Top Stroke Rehabil. 2007; $14: 5-12$.

-6 Kim CM, Eng JJ. Magnitude and pattern of 3D kinematic and kinetic gait profiles in persons with stroke: relationship to walking speed. Gait Posture. 2004;20:140-146.

7 Gutierrez EM, Bartonek A, Haglund-akerling Y, Saraste H. Centre of mass motion during gait in persons with myelomeningocele. Gait Posture. 2003;18(1):37-46.

-8 Schepers HM, van Asseldonk EHF, Buurke JH, Veltink PH. Ambulatory estimation of center of mass displacement during walking. IEEE Trans Biomed Eng. 2009;56(4):1189-1195.

-9 Shimba T. An estimation of center of gravity from force platform data. J Biomech. 1984;17(1):53-60.

10 Iida H, Yamamuro T. Kinetic analysis of the center of gravity of the human body in normal and pathological gaits. J Biomech. 1987;20(1):987-995.

11 Crowe A, Schiereck P, de Boer R, Keessen W. Characterization of gait of young adult females by means of body centre of mass oscillations derived from ground reaction forces. Gait Posture. 1993;1(1):61-68.

12 Whittle MW. Three-dimensional motion of the centre of gravity of the body during walking. Hum Move Sci. 1997; 16(1):347-355.

13 Chung SG, Hong JB, Han TR. Vertical excursion of center of gravity in relation with contra-lateral limb kinematics in poststroke hemiplegic gait. Am J Phys Med Rehabil. 2005;84(3):206.

14 Eames MHA, Cosgrove A, Baker R. Comparing methods of estimating the total body centre of mass in three-dimensions in normal and pathological gaits. Hum Move Sci. 1999;18(1):637-646.

15 Massaad F, Lejeune TM, Detrembleur C. Reducing the energy cost of hemiparetic gait using center of mass feedback: a pilot study. Neurorehabil Neural Repair. 2010;24(4):338-347.
16 Whittle MW. Three-dimensional motion of the centre of gravity of the body during walking. Hum Move Sci. 1997;16(1): $347-355$.

17 Tesio L, Lanzi D, Detrembleur C. The 3-D motion of the centre of gravity of the human body during level walking. I. Normal subjects at low and intermediate walking speeds. Clin Biomech. 1998;13(2):77-82.

- 18 Dierick F, Lefebvre C, Hecke A, Detrembleur C. Development of displacement of centre of mass during independent walking in children. Dev Med Child Neurol. 2004;46(1):533-539.

19 Detrembleur C, Hecke A, Dierick F. Motion of the body centre of gravity as a summary indicator of the mechanics of human pathological gait. Gait Posture. 2000;12:243-250.

20 Bronstrom E, Ortqvist M, Akerlind-Haglund Y, Hagelberg S, Farewik-Gutierrez E. Trunk and center of mass movements during gait in children with juvenile idiopathic arthritis. Hum Move Sci. 2007;26(1):296-305.

21 Fugl-Meyer AR, Jaasko L, Leyman I, Olsson S, Steglind S. The post-stroke hemiplegic patient: a method for evaluation of physical performance. Scand J Rehabil Med. 1975;7(1):13-31.

-22 Myiamoto ST, LombardI JI, Berg KO, Ramos IR, Natour J. Brazilian version of the Berg Balance Scale. Braz J Med Biol Res. 2004;37(8):1411-1421.

23 Bohannon RW, Smith MB. Interrater reliability of a modified Ashworth scale of muscle spasticity. Phys Ther. 1987;67(2): 206-207.

24 Barros RML, Brenzikofer R, Leite NJ, Figueroa PJ. Desenvolvimento e avaliação de um sistema para análise tridimensional de movimentos humanos. Rev Bras Eng Biomed. 1999;15(1): 79-86.

-25 Barros RML, Russomano TG, Brenzikofer R, Figueroa PJ. A method to synchronize video cameras using the audio band. J Biomech. 2006;39(4):776-780.

26 Figueroa PJ, Barros RML. A flexible software for tracking of markers used in human motion analysis. Comput Meth Prog Biol. 2003;72:155-165.

27 Cappozzo A, Catan F, Della Croce U, Leardini A. Position and orientation in space of bones during movement: anatomical frame definition and determination. Clin Biomech. 1995;10(4): 171-178.

28 Bell AL, Pedersen DR, Brand RA. A comparison of the accuracy of several hip center location prediction methods. J Biomech. 1990;23(6):617-621.

-29 Meskers CGM, van der Helm FCT, Rozendaal LA, Rozing PM. In vivo estimation of the glenohumeral joint rotation center from scapular bony landmarks by linear regression. J Biomech. 1998; 31(1):93-96.

30 Zatsiorsky VM, Seluyanov V, Chugunova L. In vivo body segment inertial parameters determination using a gammascanner method. Biomechanics of Human Movement Application in Rehabilitation, Sports and Ergonomics. Worthington, OH: Bertec Corporation; 1990; p. 186-202.

- 31 de Leva P. Adjustments to Zatsiorsky-Seluyanov's segment inertia parameters. J Biomech. 1996;29(9):1223-1230.

32 Perry J. Gait Analysis - Normal and Pathological Function. Thorofare, NJ: SLACK Incorporated; 1992.

- 33 Orendurff MS, Segal AD, Klute GK, Berge JS, Rohr ES, Kadel NJ. The effect of walking speed on center of mass displacement. J Rehabil Res Dev. 2004;41(6a):829-834.

- 34 Detrembleur C, Dierick F, Stoquart G, Chantrain EF, Lejeune T. Energy cost, mechanical work, and efficiency of hemiparetic walking. Gait Posture. 2003;18(1):47-55. 